\title{
Effect of Electron Flux on the Electronic-excitation-induced Phase Separation in GaSb
}

\section{Nanoparticles}

\author{
H. Yasuda ${ }^{*}$, A. Tanaka*, K. Ishiji ${ }^{*}$ and H. Mori ${ }^{* *}$ \\ *Department of Mechanical Engineering, Kobe University, Rokkodai, Nada, Kobe 657-8501, Japan \\ ${ }^{* *}$ Research Center for Ultra-High Voltage Electron Microscopy, Osaka University, Yamadaoka, Suita, Osaka \\ 565-0871, Japan
}

Recently, it was found that GaSb nanoparticles excited by $75 \mathrm{keV}$ electrons transform to two phases consisting of an antimony core and a gallium shell [1]. This result suggests that long-range atomic diffusion was induced by electronic excitation to separate deferent two phases, because in an isolated compound nanoparticle which has a high ratio of surface to volume, the excited states which instabilize the hetero-bond tend to localize and atomic mobility is higher than that in the corresponding bulk materials. In the present work we have studied the effect of electron flux on the phase separation induced by electronic excitation in GaSb nanoparticles.

Preparation of size-controlled GaSb particles was carried out with the use of a double-source evaporator installed in the specimen chamber of an electron microscope. Electronic excitation experiments and observations were carried out using the same microscope. The microscope used was Hitachi H-800 transmission electron microscope operating at an accelerating voltage of $75 \mathrm{kV}$. The electron fluxs used for excitations were from $5.0 \times 10^{20}$ to $5.0 \times 10^{21} \mathrm{e} \mathrm{m}^{-2} \mathrm{~s}^{-1}$. The temperature of the supporting films was kept at $423 \mathrm{~K}$ during the experiments. Phase separation associated with electronic excitations was observed in situ by bright-field images (BFIs) and selected-area electron diffraction patterns (SAEDs).

An example of the structural change in GaSb particles by electronic excitation with $75 \mathrm{keV}$ incident electrons at the flux of 5.0x10 $10^{21} \mathrm{e} \mathrm{m}^{-2} \mathrm{~s}^{-1}$ is shown in Fig. 1. Figures 1(a) and (a') show a BFI of particles with the diameter of 10-20 $\mathrm{nm}$ before excitation and the corresponding SAED, respectively. As indexed in the figure $\left(1\left(\mathrm{a}^{\prime}\right)\right)$, the Debye-Scherrer rings can be consistently indexed as those of GaSb which has the zincblende structure with a lattice constant of $\mathrm{a}_{0}=0.61 \mathrm{~nm}$. The same area after excitation for $90 \mathrm{~s}$ (i.e., up to the dose of $4.5 \times 10^{23} \mathrm{e} \mathrm{m}^{-2}$ ) is shown in Fig. 1(b). In the interior of approximately $10 \mathrm{~nm}$-sized particles after the excitation, there appears a structure consisting of a core with dark contrast and a shell with bright contrast, as seen from a comparison of the part framed squarely by $\mathrm{I}_{\mathrm{a}}$ in (a) with that framed by $\mathrm{I}_{\mathrm{b}}$ in (b). The SAED taken after the excitation is shown in Fig. 1(b'). In the SAED, Debye-Scherrer rings are recognized, superimposed on a weak halo ring. The Debye-Scherrer rings can be indexed consistently as those of crystalline antimony, which has the hexagonal structure with lattice constants of $\mathrm{a}_{0}=0.43 \mathrm{~nm}$ and $\mathrm{c}_{0}=1.13 \mathrm{~nm}$, and of $\mathrm{GaSb}$. The value of the scattering vector $(\mathrm{K}=(4 \pi \sin \theta) / \lambda)$ for the halo ring is approximately $31.0 \mathrm{~nm}^{-1}$ which is corresponding to the first halo of liquid gallium. The same area after excitation for $180 \mathrm{~s}$ (i.e., up to the dose of $9.0 \times 10^{23} \mathrm{e} \mathrm{m}^{-2}$ ) is shown in Fig. 1(c). Also in the interior of approximately $20 \mathrm{~nm}$-sized particles after the excitation, there appears a structure consisting of a core with dark contrast and a shell with bright contrast, as seen from a comparison of the parts framed by $\mathrm{II}_{\mathrm{a}}$ in (a) and $\mathrm{II}_{\mathrm{b}}$ in (b) with the part framed by $\mathrm{II}_{\mathrm{c}}$ in (c). In the SAED shown in Fig. 1(c'), Debye-Scherrer rings of crystalline antimony are recognized, superimposed on a halo ring of liquid gallium. It was confirmed by 
dark-field electron microscopy that nanoparticles after the electronic excitation have the two-phase structure consisting of a crystalline antimony core and a liquid gallium shell. From these results, it is evident that two-phase separation takes place in GaSb particles excited at the flux of 5.0x10 $0^{21} \mathrm{e} \mathrm{m}^{-2} \mathrm{~s}^{-1}$. On the other hand, it was confirmed that such a phase separation as observed after electronic excitation at the flux of $5.0 \times 10^{21} \mathrm{e} \mathrm{m}^{-2} \mathrm{~s}^{-1}$ is absent during the electronic excitation at lower flux of 5.0 $\mathrm{x}^{20} 0^{20} \mathrm{e} \mathrm{m}^{-2} \mathrm{~s}^{-1}$. Through the experiments, it has been evident that the phase separation induced by electronic excitation with $75 \mathrm{keV}$ incident electrons in $\mathrm{GaSb}$ particles kept at $423 \mathrm{~K}$ becomes more difficult to occur with decreasing electron flux. The behaviors of the electronic-excitation-induced phase separation will be discussed as functions of the electron flux as follows.

Figure 2 shows the change in the rate of the phase separation per unit dose as a function of the flux. At the flux of $5.0 \times 10^{20} \mathrm{e} \mathrm{m}^{-2} \mathrm{~s}^{-1}$, the phase separation does not take place at all, however at the flux of $1.0 \times 10^{21}$ or $5.0 \times 10^{21} \mathrm{e}$ $\mathrm{m}^{-2} \mathrm{~s}^{-1}$, the phase separation proceeds at the rate of the phase separation per unit dose of $6.1 \times 10^{-25}$ and $8.4 \times 10^{-25}(\mathrm{e}$ $\left.\mathrm{m}^{-2}\right)^{-1}$, respectively. It is evident from the figure that a fixed and higher flux is required for the electronic-excitation-induced phase separation and the rate of the phase separation per unit dose becomes nearly constant at the flux above the threshold value (the range from $5.0 \times 10^{20}$ to $1.0 \times 10^{21} \mathrm{e} \mathrm{m}^{-2} \mathrm{~s}^{-1}$ in this case).

The phase separation proceeds abruptly after an incubation time with increasing electron dose and does only at the flux above a threshold value. Such nonlinear behaviors suggest that the synergistic effect among electronic-excitation effect, nano-size effect and temperature plays an important role to achieve stabilization of the homo-bonds and enhancement of long-range atomic diffusion required for the electronic-excitation-induced phase separation.

\section{References}

[1] H.Yasuda, H.Mori and, J. G. Lee. ,Phys. Rev. Lett. 92, 135501 (2004).
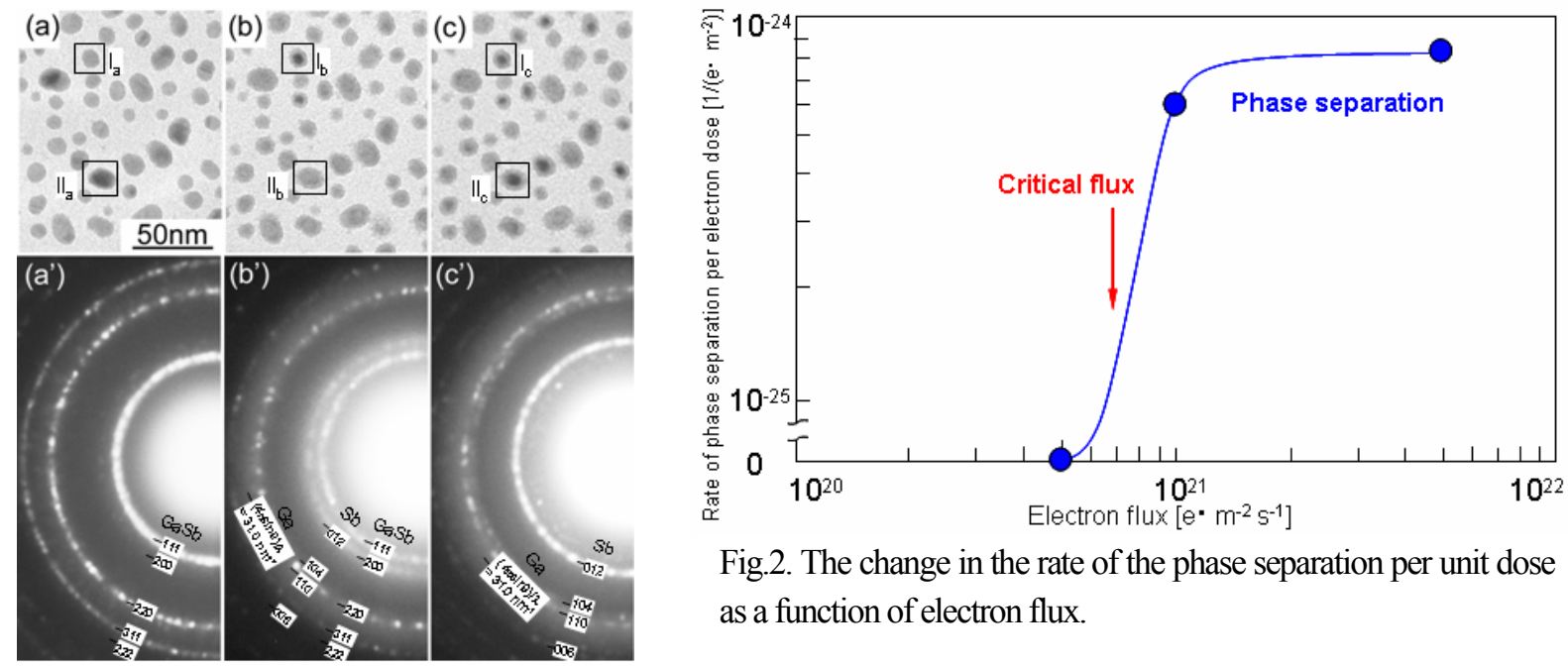

Fig.2. The change in the rate of the phase separation per unit dose as a function of electron flux.

Fig. 1. An example of the structural change in GaSb particles by $75 \mathrm{keV}$ electronic excitation at the flux of 5.0x $10^{21} \mathrm{e} \mathrm{m}^{-2} \mathrm{~s}^{-1}$. (a) a BFI, and (a') the corresponding SAED before excitation. (b) after excitation for $90 \mathrm{~s}$, and (b') the corresponding SAED. (c) after excitation for $180 \mathrm{~s}$, and (c') the corresponding SAED. 